\title{
Differential forms of p53 in medulloblastoma primary tumors, cell lines and xenografts
}

\author{
TZVETOMIRA PHILIPOVA ${ }^{1}$, NINIB BARYAWNO ${ }^{2}$, WOLFGANG HARTMANN ${ }^{4}$, TORSTEN PIETSCH $^{5}$, \\ HENRIK DRUID $^{3}$, JOHN INGE JOHNSEN ${ }^{2}$ and TOMAS J. EKSTRÖM ${ }^{1}$ \\ Departments of ${ }^{1}$ Clinical Neuroscience, Center for Molecular Medicine, Karolinska University Hospital, L8:01, \\ ${ }^{2}$ Women's and Children's Health, Childhood Cancer Research Unit, ${ }^{3}$ Oncology-Pathology, Forensic \\ Medicine, Karolinska Institutet, S-171 76 Stockholm, Sweden; Departments of ${ }^{4}$ Pathology, \\ and ${ }^{5}$ Neuropathology, University of Bonn Medical Center, D-53127 Bonn, Germany
}

Received September 21, 2010; Accepted November 1, 2010

DOI: $10.3892 /$ ijo.2010.884

\begin{abstract}
Medulloblastoma is the most common malignant brain tumor in childhood. The most prevalent chromosomal abnormalities are isochromosome $17 \mathrm{q}$ and loss of $17 \mathrm{p}$, the location of the tumor suppressor gene p53. Mutations in the p53 gene in medulloblastoma are relatively infrequent but have recently been correlated to poor prognosis. Furthermore, the p53 gene encodes nine different isoforms, which may have a profound impact on p53 tumor suppressor activity. Nine medulloblastoma primary biopsy samples, six cell lines from medulloblastoma, and one from a supratentorial PNET, and a medulloblastoma xenograft, along with human brain and visceral tissues, were analyzed by Western blotting, using monoclonal p53 antibodies against two regions in the Nterminus or the central domain. Medulloblastoma primary tissue and xenografts present low molecular weight proteins recognized by both $\mathrm{N}$-terminal p53 antibodies that are absent in all cell lines including the one used for xenografts. Normal visceral organs display short forms of p53, and low levels of canonical p53. Normal brain structures, including cerebellum, contained only canonical size p53 at high levels. In conclusion, our results indicate that the presence of p53 isoforms may play a functional role in medulloblastoma. The observed differences in their presence in cell lines and derived xenografts, suggest that $\mathrm{p} 53$ should be investigated in in vivo models rather than in cell lines.
\end{abstract}

\section{Introduction}

Medulloblastoma (MB) is the most common malignant brain tumor in children. The tumor, and the current treatment

Correspondence to: Dr Tomas Ekström, Department of Clinical Neuroscience, Karolinska Institutet, Center for Molecular Medicine, Karolinska University Hospital, L8:01, S-171 76 Stockholm, Sweden E-mail: tomas.ekstrom@ki.se

Key words: medulloblastoma, p53, N-terminus, p53 isoforms, Western blot analysis modalities, in particular surgery and irradiation (frequently in combination with chemotherapy), cause adverse effects to the majority of long-term survivors. MB therefore represents an important therapeutic challenge which requires novel approaches based on current biological knowledge.

The WHO classifies 5 different variants of MB based on histopathological characteristics (1). The treatment of MB is performed by surgical resection, craniospinal radiation and chemotherapy. The 5-year progression-free survival after treatment is around $80 \%$ (2). However, even if the morbidity due to the aggressive therapy gradually has been reduced, it is still a substantial problem (3).

Together with clinical factors (e.g. subtotal tumor resection) there are molecular features of the tumor, like tyrosine kinase receptor ERBB2 overexpression and loss of genetic material at chromosome $17 \mathrm{p}$ that indicate a poor chance of survival $(4,5)$. The majority of MBs are sporadic, but familial predisposition related to germline mutations exists (6). Familial MBs have assisted in the identification of relevant pathways for sporadic MB, such as in the Turcot syndrome with APC mutations involving the Wnt/Wingless pathway (7), and the Gorlin syndrome which is associated with mutations in the PTCH1 gene affecting the Sonic hedgehog pathway (8). Mutations of the TP53 tumor suppressor gene have been shown to be rare in $\mathrm{MB}$, but it was recently reported that $16 \%$ of MB have a mutated TP53 gene, and these patients were strongly associated with worse 5-year survival (9). Furthermore, appearance of an isochromosome $17 \mathrm{q}$ is frequently observed (10). TP53 is located on $17 \mathrm{p}$ but its role in the pathogenesis of $\mathrm{MB}$ is not clear, although data suggest that the p53-p14ARF pathway is involved (11). The wild-type p53 protein is a sequence-specific transcription factor that plays a central role in cancer surveillance. The p53 protein contains several functional domains: the activation domain 1 (within residues 1-42), the activation domain 2 [43-63], the proline-rich domain [64-91], the sequence specific DNA binding domain [100-300], the nuclear localization signal [316-325], the tetramerization domain [334-356], and the Cterminal domain [364-393] (12).

TP53 has been reported to occur as an N-terminal truncated version denoted $\mathrm{p} 47, \Delta 40 \mathrm{p} 53$ or $\Delta \mathrm{N}-\mathrm{p} 53$ (13-16). This protein 
is generated by the employment of an alternative translation start site. The direct consequence of this is a protein with an apparent size of $47 \mathrm{kDa}$, lacking the $\mathrm{N}$-terminal transactivation and Mdm-2 binding domain of p53, but which can form heterotetramers with full length p53 and interfere with p53 function $(13,14)$. p47 may act as a 'cell cycle facilitator' through its capacity to negatively regulate p53 transactivation. The human p53 gene can encode at least nine different p53 protein isoforms: $\mathrm{p} 53, \mathrm{p} 53 \beta, \mathrm{p} 53 \gamma, \Delta 133 \mathrm{p} 53, \Delta 133 \mathrm{p} 53 \beta$ and $\Delta 133 \mathrm{p} 53 \gamma$ are produced by alternative splicing of the intron 9 , and alternative usage of the alternative promoter in intron 4 , and $\Delta 40 \mathrm{p} 53$ (p47), $\Delta 40 \mathrm{p} 533$ and $\Delta 40 \mathrm{p} 53 \gamma$ - produced by alternative splicing of intron 9 , and alternative intitiation of translation or alternative splicing of intron 2 (14).

p53 isoforms have been described in breast cancer, acute myeloid leukemia, neuroblastoma, and in squamous cell carcinoma of the head and neck (17-21). In leukemia the appearance of a low molecular weight form of p53 is inversely correlated with the response to induction chemotherapy (17). Despite all the experimental evidence showing the importance of p53 in preventing carcinogenesis, it is difficult in clinical studies to link p53 status to cancer treatment and clinical outcome. The p53 isoforms may have profound impact on our understanding of p53 tumor suppressor activity (20). In this study we used different specific anti-p53 antibodies to evaluate p53 status and variants in patients with $\mathrm{MB}$, in cell lines and xenografts.

\section{Materials and methods}

Ethics statement. Human postmortem tissue was obtained with approved ethical permission by the Stockholm Regional Ethics Review Board. Prospective written informed consent was obtained by the donors. The HIT study protocol, including the asservation and scientific analysis of medulloblastoma tumor tissue, was approved by the ethics committee of the University of Wuerzburg. Written informed consent was obtained from legal representatives of all patients before registration.

All animal experiments were approved by the Northern Stockholm Regional Ethics Committee for animal research (approval N234/05) in accordance with the Animal Protection Law (SFS1988:534), the Animal Protection Regulation (SFS 1988:539), and the Regulation for the Swedish National Board for Laboratory Animals (SFS1988:541).

Tissue preparation. Nine samples of MB (8 classic, 1 desmoplastic) were collected at the Department of Neuropathology, University of Bonn Medical Center, Germany, and frozen at $-80^{\circ} \mathrm{C}$. The frozen sections from the MB samples contained at least $80 \%$ tumor tissue. Normal human tissue samples dissected from postmortem human brains and visceral organs were obtained at autopsy and immediately frozen. Frozen tissue samples were kept on dry ice during tissue weight measurement and crushing. Crushed, powdered tissue samples were kept at $-80^{\circ} \mathrm{C}$. Human MB xenograft samples from mice were dissected and imme-diately frozen. All samples were stored at $-80^{\circ} \mathrm{C}$.

Cell lines and cell culturing. Six MB cell lines - D283Med (22), D324Med (23), D425Med and D458Med (24), MEB-MED8A
(25), UW228-3 (26), and one supratentorial PNET, PFSk-1 (27), were used in the experiments for evaluating the p53 status by Western blotting. D283Med, D324Med, D425Med, D458Med and MEB-MED8A cell lines were maintained in Dulbecco's modified Eagle's medium (DMEM, Invitrogen), supplemented with $10-20 \%$ of fetal bovine serum (FBS) and antibiotics (100 units $/ \mathrm{ml}$ penicillin - $100 \mu \mathrm{g} / \mathrm{ml}$ streptomycin) in an atmosphere of $5 \%$ of $\mathrm{CO}_{2}$. PFSk-1 was maintained in RPMI and UW228-3 in DMEM:F12 (Invitrogen), supplemented with $10-20 \%$ of FBS and antibiotics (100 units $/ \mathrm{ml}$ penicillin - $100 \mu \mathrm{g} / \mathrm{ml}$ streptomycin) in an atmosphere of $5 \%$ of $\mathrm{CO}_{2}$.

Xenografts. Subcutaneous MB xenografts were obtained by transplanting the human D283Med MB cells in mice. Four to eight-week old female NMRI nu/nu mice (Taconic Laboratories, Ejby, Denmark) were maintained in groups of 5 per cage and were given sterile water and food ad libitum. Each NMRI nu/nu mouse was s.c. injected in the flank with $20 \times 10^{6}$ D283MED MB cells. Tumors were measured every day and tumor volume was calculated as (width) ${ }^{2} \mathrm{x}$ length $\mathrm{x} 0.44$. After 9 days, the tumor volume was approximately $0.2 \mathrm{ml}$.

Plasmid and transfection. Positive control for full-length p53 was produced by transfecting a p53 expression plasmid into Hep3B cells, which are negative for $\mathrm{p} 53$. The same cell line was used for producing the p53 N-terminal truncated p47 positive control. The p53 and p47 expression plasmids were kind gifts from Dr Pierre Hainaut, Lyon, France. Hep3B cells were passaged in DMEM, $10 \%$ FBS, $1 \%$ antibiotics to $60-70 \%$ confluence before transfection. The cells were transfected with either a p53 or p47 expression plasmids using Lipofectamine Plus in Opti-MEM medium (Invitrogen) according to the manufacturer's recommendations.

Preparation of cell/tissue extracts. Buffers were supplemented with $5 \mathrm{X}$ protease inhibitors, Complete ${ }^{\mathrm{TM}}$, (Boehringer Mannheim) and $5 \mathrm{mM}$ of the proteasome inhibitor MG132 (Calbiochem). All procedures were carried out on ice. Frozen tissue samples were crushed on dry ice and boiled in prewarmed SDS extraction buffer $(0.45 \mathrm{M}$ Tris- $\mathrm{HCl}, \mathrm{pH} 8.5$, $2.5 \%$ glycerol, $4 \%$ SDS, $0.5 \mathrm{mM}$ DTT) and $5 \mathrm{X}$ protease inhibitors for $5 \mathrm{~min}$, sonicated, centrifuged, and protein extracts were aliquoted and kept at $-80^{\circ} \mathrm{C}$ until use. Protein concentration was determined with the DC protein assay (Bio-Rad Laboratories). Aliquots of tissue extracts (25-60 $\mu \mathrm{g}$ protein) were heated for $5 \mathrm{~min}$ at $+95^{\circ} \mathrm{C}$ in the presence of $5 \mathrm{mM} ß$-mercaptoethanol and separated by SDS PAGE on $10 \%$ Tricine gels.

Western blot analysis. Equal amounts of cell/tissue protein extracts were separated by SDS-PAGE on 10\% Tricine large $(13 \times 15 \mathrm{~cm})$ gels (Acrylamide, Gel buffer, 100\% Glycerol, $\mathrm{dH}_{2} \mathrm{O}, 10 \%$ ammonium persulfate, TEMED, Bio-Rad Laboratories). Proteins were electrotransferred onto a nitrocellulose membrane (Schleicher and Schuell) at $4^{\circ} \mathrm{C}$ and stained by MemCode Reversible Protein Stain kit (Pierce). After destaining and blocking with 5\% non-fat dry milk in Trisbuffered Saline Tween-20 (TBST), the membranes were incubated with monoclonal anti-p53-antibodies directed against 


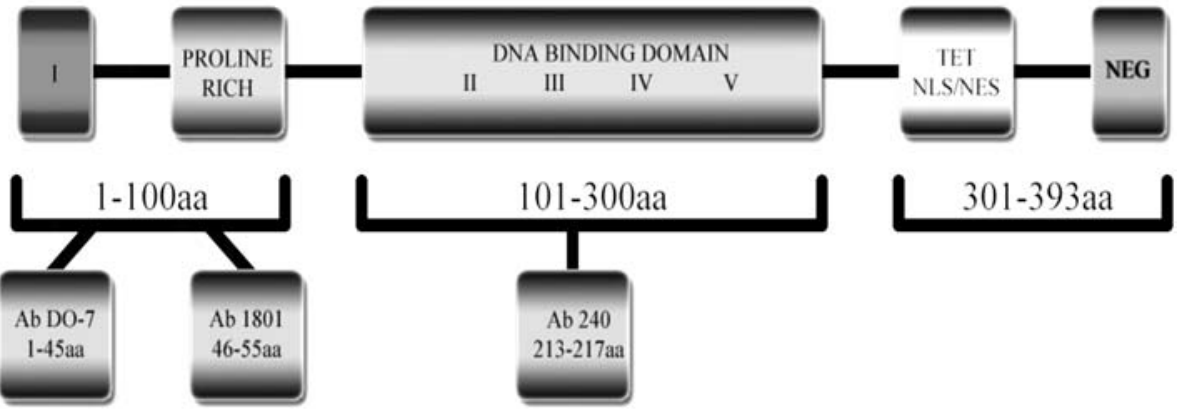

Figure 1. Human p53 protein structure and antibodies used in the present study. The extreme N-terminus (1-42aa) contains the acidic transactivation domain and the MDM2 protein binding site. It also contains the Highly Conserved Domain I (HCD I). Region 40-92aa contains series of proline residues that are less evolutionary conserved, and a second transactivation domain. The central region (101-306aa) - the target of $90 \%$ of p53 mutations found in human cancers contains the DNA binding domain. It contains HCD II to V. The oligomerization domain (307-355aa, TET) consists of a $\beta$-strand, followed by an $\alpha$-helix necessary for dimerization. This oligomerization domain also contains a nuclear export signal (NES). The C-terminus of p53 (356-393aa) contains 3 nuclear localization signals (NLS) and a non-specific DNA binding domain that binds to damaged DNA. This region is also involved in the downregulation of DNA binding of the central domain. NEG is the C-terminal negative regulatory domain.

the p53 N-terminus: Ab1801 (1:50 dilution, Calbiochem) and AbDO-7 (1:250 dilution, Dako), or the p53 central domain, Ab240 (1:80 dilution, Calbiochem). As a cytosolic marker, a monoclonal antibody against $\gamma$-enolase was used (NSE-P2, 1:1000, Santa Cruz Biotechnology). The membranes were washed with Tris-Buffered Saline Tween-20 (TBST) and subsequently probed with a horseradish peroxidase conjugated goat anti-mouse immunoglobulin antibody (Calbiochem) at 1:2000 dilution, developed by ECL (Amersham) according to the manufacturer's instructions and exposed for 1-10 min on Hyperfilm-ECL (Amersham).

For reprobing, membranes were incubated with stripping buffer (62.5 mM Tris- $\mathrm{HCl}, 2 \%$ SDS, $100 \mathrm{mM} \beta$-mercaptoethanol, $\mathrm{pH}$ 6.7) for $20 \mathrm{~min}$ at $55^{\circ} \mathrm{C}$, blocked for $30 \mathrm{~min}$ with $5 \%$ non-fat dry milk in $50 \mathrm{mM}$ Tris- $\mathrm{HCl}, 0.15 \mathrm{M} \mathrm{NaCl}$, $0.05 \%$ Tween-20 buffer, and probed with the goat anti-mouse immunoglobulin antibody (dilution 1:2000). Quantification of Western blots was performed by phosphoimager using Image Gauge Ver.3.11 software (Fuji Photo Film Co. Ltd., Japan).

\section{Results}

The expression of different p53 isoforms in human cancers may explain the inconsistencies in the published findings on the prognostic role of $\mathrm{p} 53$. The finding that the $p 53$ gene can produce a number of different transcripts prompted us to investigate the presence of protein variants of p53 in solid tumors and to compare this with human brain regions and visceral tissues. This was done by analyzing a number of tumor samples using Western blot with three different antibodies specific for different domains of the protein (Fig. 1). AbDO-7 recognizes aa 1-45 in the extreme N-terminus, Ab1801 recognizes aa 46-55 in the N-terminus, and Ab240 binds to aa 213-217 in the central domain.

p53 variants vary in medulloblastoma tumors and xenografts. The expression of p53 in clinical MB samples, cell lines and xenografts was examined by Western blot using antibodies against the N-terminus of p53. Detection of a band consistent with the expected full length canonical MW of wild-type p53

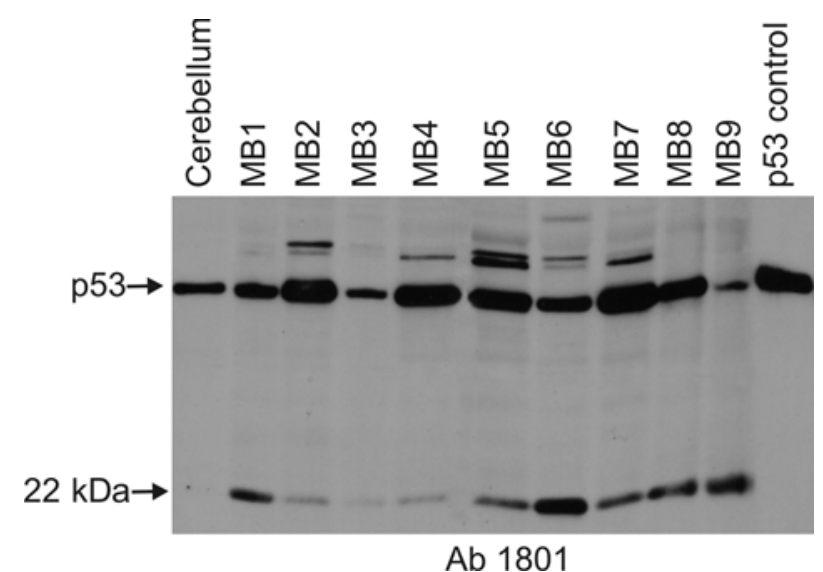

Figure 2. p53 status in medulloblastoma (MB) samples, detected by Ab1801. p53 expression was analyzed in cerebellum and MB samples from 9 different subjects and protein extracts $(50 \mu \mathrm{g})$ were loaded on $10 \%$ SDS-PAGE high resolution gels. Arrows indicate full-length p53 (upper arrow) and its short form $\sim 22 \mathrm{kDa}$ (lower arrow) as detected by antibody Ab1801. Positive control for full-length p53 was produced by transfecting a p53 expression plasmid into the p53 deficient Hep3B cell line.

(FL) was found in the majority of the samples (Fig. 2) using the antibody Ab1801 (directed to aa 46-55). The expression level of canonical p53 was variable between the different MB primary tumor samples. In addition, a protein species with an apparent size of $22 \mathrm{kDa}$ was present at varying levels in all MB tumors. The levels of the $22 \mathrm{kDa}$ protein did not proportionally correlate with the amount of the FL p53 (Fig. 2). Neither the control p53 generated from the transfected p53 canonical plasmid construct, nor the fetal cerebellum displayed the $22 \mathrm{kDa}$ protein. In some of the MB samples, variable amounts of larger species of p53 with unknown function were also apparent (Fig. 2).

Since MB samples contain heterogeneous cell populations, we also analyzed the p53 pattern in 7 different MB cell lines. Using Ab1801 and AbDO-7 N-terminus-specific antibodies, the MB cell lines displayed p53 with variable sizes around $53 \mathrm{kDa}$ (Fig. 3). Since the N-truncated p47 isoform was 


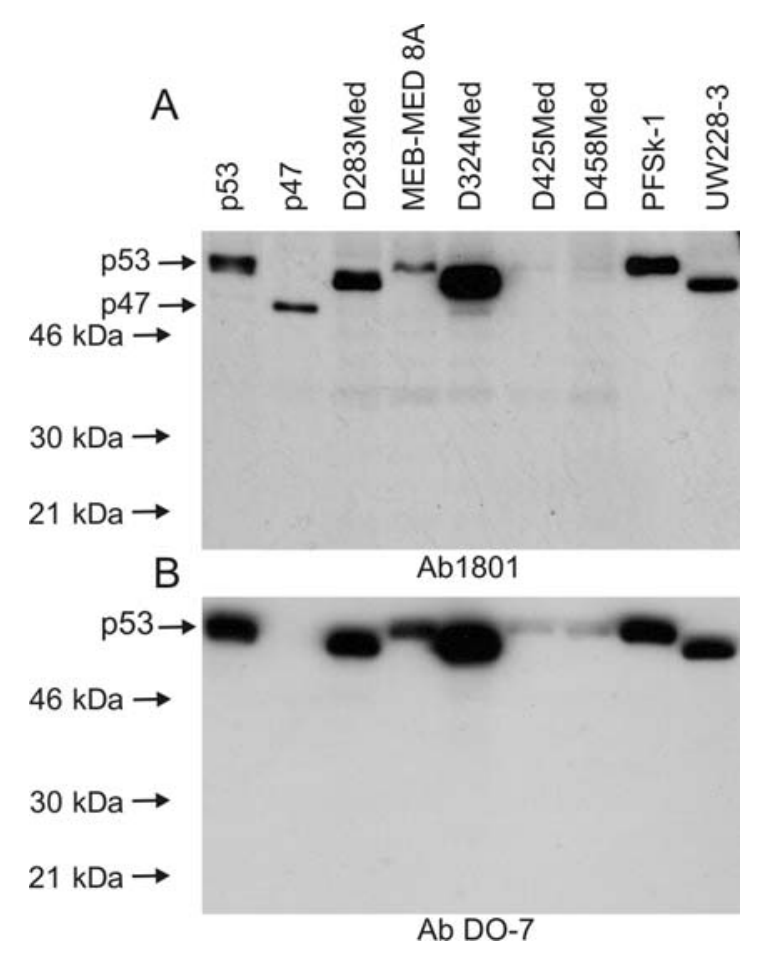

Figure 3. p53 status in medulloblastoma cell lines, detected by Ab1801 and AbDO-7. p53 expression was analyzed in 6 different human medulloblastoma cell lines: D283Med, MEB-MED8A, D324Med, D425Med, D458Med, UW228-3 and one supratentorial PNET, PFSk-1. Protein extracts were loaded on $10 \%$ SDS-PAGE high-resolution gels. Arrows indicate fulllength p53 and p47 detected by Ab1801, (A). The same pattern of p53 expression was detected by using AbDO-7, (B). Positive controls for fulllength p53 and for N-terminal truncated p47 proteins were produced by transfecting a p53 and a p47 expression plasmid, respectively, into the p53 deficient Hep3B cell line.

previously reported as being a regulatory factor for $\mathrm{p} 53$, we transfected the p53 negative Hep3B cells with p47 in an expression vector and included this in the panel of MB cell lines. Western blot analysis revealed that the Ab1801 antibody is able to recognize p47, while the AbDO-7, which is directed to the very end of the N-terminus of $\mathrm{p} 53$, does not (Fig. 3A). This served as a control for the $\mathrm{p} 47$ truncation being only in the first 45 amino acids of the N-terminus of p53. The various sizes of p53 in MB cell lines appear to be very similar irrespective of antibody used (Ab1801 or AbDO-7; Fig. 3). We therefore conclude that truncation of the very $\mathrm{N}$-terminus does not explain the variable and shorter p53 species in MB cell lines. No $22 \mathrm{kDa}$ species of p53 were detected in MB cell lines.

In order to investigate whether the tumor growth environment of MB might affect the p53 isoforms, the cell line D283Med that displayed a p53 species with a slightly smaller apparent migration than FLp53 when grown in culture, was implanted into nude mice to generate subcutaneous xenografts. Western blot analysis of the xenografts, using the Ab1801 antibody, revealed predominantly a protein that corresponds to FLp53. In addition to this, a weak band corresponding to $22 \mathrm{kDa}$ was also evident (Fig. 4). Thus, a smaller p53 protein isoform was present, albeit less abundant than FL p53, both in MB tumor samples (Fig. 2) and in xenografts (Fig. 4) obtained from a cell line (Fig. 3) which did not express this

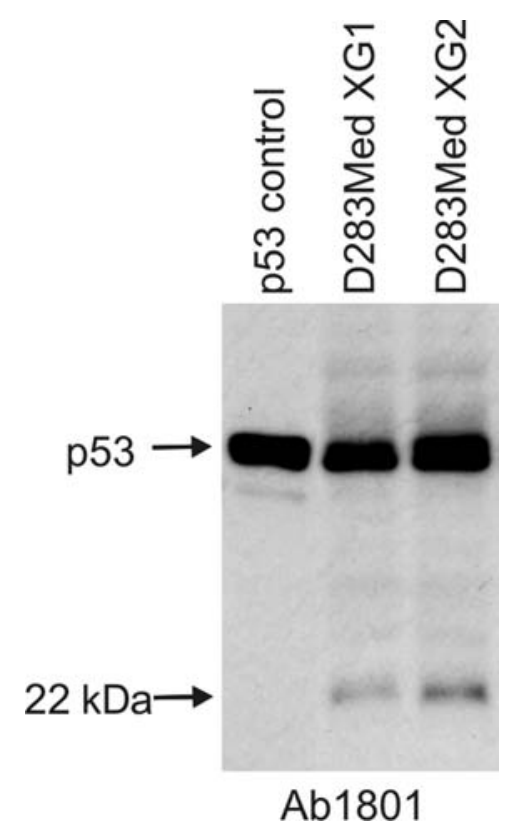

Figure 4. p53 status in medulloblastoma xenografts using Ab1801. p53 expression was analyzed in medulloblastoma xenografts on $10 \%$ SDSPAGE high resolution gels. Subcutaneous xenografts were obtained from the human MB cell line D283Med transplanted into nude mice. Arrows indicate full-length p53 and its short form $22 \mathrm{kDa}$ detected by PAb1801. Positive control for full-length p53 was produced by transfecting a p53 expression plasmid into Hep3B cells.

isoform. Thus, the p53 protein is produced in isoform(s) in $\mathrm{MB}$ tumor tissue and xenografts, not observed in the corresponding MB cell lines.

p53 isoforms in normal tissues. As the p53 isoforms might have different biological activities, we investigated whether p53 isoforms are differentially expressed in normal human tissues as was observed in MB. Human brain tissue from cerebellum and temporal cortex, which both revealed only FLp53, was compared with different human organs, heart, lung, liver, intestine and testis using the N-terminus-specific Ab1801 and the central DNA-binding domain-specific Ab240 antibodies. In normal visceral human organs, several short forms of p53 were detected using both antibodies (Fig. 5A and B). The expression of the low molecular weight forms was variable between the different human tissues and was often coexpressed with the FLp53. In lung tissue, the two antibodies gave similar band patterns with two low molecular weight bands, while in heart, intestine, liver and testis, only one of the low molecular bands was visible using Ab1801 (Fig. 5). In visceral tissues, FLp53 was generally expressed at low levels.

Subcellular localization of p53 in brain tissue. The nuclear localization of canonical p53 is essential for its tumor suppressor function. The p53 protein may, however, have different subcellular localizations and therefore distinct activities. In order to investigate this in the normal human brain, we analyzed nuclear and cytosolic fractions of cerebellum since this is the origin of $\mathrm{MB}$, and temporal cortex, as a brain control tissue. These brain structures were assessed with Ab1801, using $\gamma$-enolase as a cytosolic neuronal 

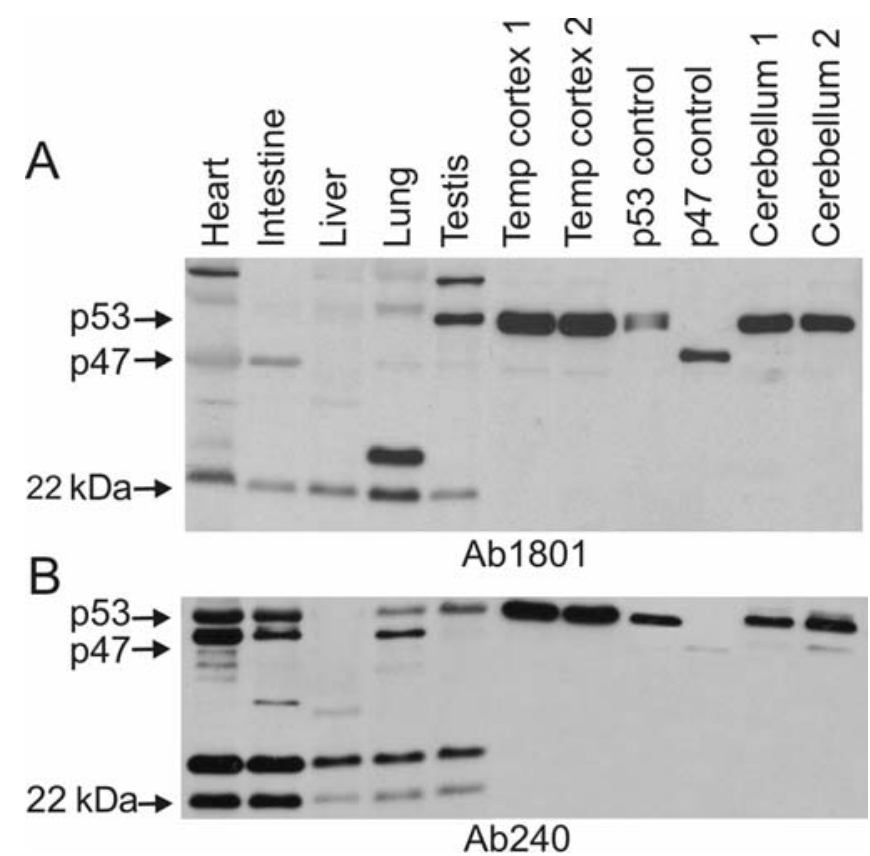

Figure 5. p53 status in human tissues from visceral organs and brain structures. p53 expression was analyzed in 5 visceral organs, and in temporal cortex and cerebellum from 2 different subjects. Protein extracts were loaded on $10 \%$ SDS-PAGE high-resolution gels. Arrows from top to bottom indicate full-length $\mathrm{p} 53$ protein, truncated $\mathrm{p} 47$ form and the low molecular weight form ( 22 kDa) detected by Ab1801 (A), and Ab240 (B). Positive controls for full-length $\mathrm{p} 53$ and for $\mathrm{N}$-terminal truncated $\mathrm{p} 47$ were produced by transfecting a p53 and a p47 expression plasmid into the p53 deficient Hep3B cell line.

marker. Two patterns were found: in the temporal cortex, p53 was completely absent in the cytosol, while cerebellum contained the p53 protein in both the nucleus and cytosol (Fig. 6). MB tissues could not be used for subcellular analysis.

\section{Discussion}

As p53 isoforms may bind differentially to promoters, modulate p53 target gene expression and p53-mediated apoptosis, and provide regulatory effects of canonical p53, this can explain the difficulties to link p53 status to the biological properties and drug sensitivity of human cancer (20). The aim of the present study was to characterize the p53 isoform status in MB in comparison with normal brain and visceral tissues. Unlike many other cancers, MB cells usually express wild-type p53 and mutational inactivation is relatively rare. We report here the presence of low molecular weight p53 forms (LMWFs) in MB samples, both in solid tissue tumors and in MB xenografts (Fig. 2 and 4). Since none of the tested MB cell lines displayed LMWF p53 (Fig. 3), it is possible that truncated forms, e.g. the $22 \mathrm{kDa}$ variant found in tumors and xenografts, might be produced in non-tumor cells surrounding the tumor cells. Alternatively, variants of p53 may be present in the same cell as the canonical p53, induced by the tumor environment. Although this has not been addressed in this study, the presence of short forms might lead to interactions that could affect its biological activity as was shown for the truncated p53 form, p47, which act in a

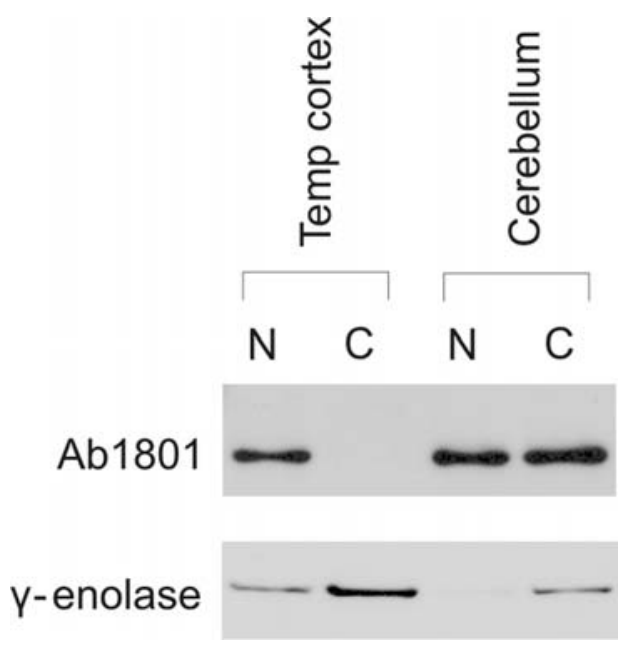

Figure 6. p53 subcellular localization in human brain structures, detected by Ab1801. Nuclear (N) and cytosolic (C) fractions of cerebellum and temporal cortex were assessed for p53 subcellular localization using the Ab1801 p53 antibody, and anti $\gamma$-enolase as a cytosolic neuronal marker.

dominant-negative manner towards FLp53, both inhibiting p53 transcriptional activity and p53-mediated apoptosis $(14,20)$. The expression of various isoforms may therefore partly account for aberrant functions of p53 in $\mathrm{MB}$, as was shown in melanoma where $440 \mathrm{p} 53$ and $\mathrm{p} 53 \beta$ were shown to form complexes with p53 (28). The proportion of p53 isoforms relative to the wild-type protein may thus be important for regulating p53-dependent transcription in MB. p53 protein isoforms are less abundant than FL p53 both in MB solid tissue samples and xenografts. However, p53 undergoes multiple post-translational modifications $(29,30)$ and specific modifications may be targeted to distinct p53 isoforms to give specific biological effects. In addition to low molecular weight form p53, modifications leading to larger p53 species are indeed suggested in this study, both in MB tumor tissue as well as in visceral organ tissue (Figs. 2 and 5). As in many other tumors, MB is formed by a heterogeneous population of cells that may express p53 at various levels in different cells, and the ratio between FLp53 and its variants might vary as well. In MB cell lines, which can be considered substantially more homogeneous, p53 LMWFs were not detected, although some variability around $53 \mathrm{kDa}$ was evident (Fig. 3). Some of these forms displayed slightly smaller apparent size than $53 \mathrm{kDa}$. Since even the antibody AbDO-7, which specifically recognizes the $\mathrm{N}$ terminus (1-45 aa), recognizes these molecular forms, any presence of smaller sizes must be accounted for by either Cterminus truncation or differential splicing, leading to faster migration on the gel of the protein product. None of the MB cell lines displayed a form co-migrating with $\mathrm{p} 47$.

In the normal human tissues, different p53 forms were found in a tissue-dependent manner (Fig. 5), suggesting that the internal promoter/alternative splicing of p53 can be tissuespecifically regulated. This may lead to different transcriptional activity in response to different types of cellular stress. It is also notable that in some tissues (e.g. liver, lung, intestine, heart, testis) the protein levels of the LMWFs exceed that of the FLp53. With the exception of heart and 
intestine, in visceral tissues FLp53 was expressed at relatively low levels, consistent with p53 being expressed at low levels in normal cell unless activated and stabilized by DNA damage (31). We also noted that in the intestine, liver and lung, FLp53 was not detected by the Ab1801 antibody, and in the liver also Ab240 did not recognize FLp53. We do not know the reason for this but it is possible that tissuespecific modifications in the $\mathrm{N}$-terminus epitope prevent Ab1801 from binding. Degradation is not likely since both the intestine and lung display FLp53 using Ab240. The complex p53 patterns in visceral tissues (Fig. 5) underscore the need for more thorough investigations using several antibodies as well as functional studies.

FLp53 is multifunctional and is found as a nuclear protein (20), but may enter the cytosol during cellular stress (32-35). If p53 isoforms have different subcellular localizations, this might be relevant for their various biological activities. LMWF of p53 were indeed shown to be abnormally localized in melanoma, where the endogenously expressed p53 isoforms may have differential localization between the nucleus and the cytosol of melanoma cells, compared with the wild-type p53 (28). The different p53 subcellular localization shown in the present study, even between the two different brain structures, cerebellum and temporal cortex (Fig. 6), may reflect differences in protective mechanisms in the different parts of the brain which may be involved in the pathogenesis of MB.

Taken together, our results suggest that the presence of p53 isoforms and their proportion to the expression of the full-length protein may be involved in regulating p53dependent transcription. One important conclusion of this study is that since differences in the presence of p53 isoforms were found between cell lines and their derived xenografts as well as with MB tissue, it is suggested that p53 should be investigated in in vivo models rather than in vitro in cell lines.

The selection of antibodies to be applied in quantitative p53 assays is important to assess various p53 isoforms, the proportion of which may hint at biological activity. This selection may establish specificity for subsequent clinical prognosis. The prediction of outcome in MB would permit allocation of patients with poor survival prognosis to appropriate aggressive therapeutic regimen, while reducing therapeutic morbidity in patients with better prognosis.

\section{Acknowledgements}

This study was supported by the Children's Cancer Foundation of Sweden, the Swedish Cancer Society and funds from the Karolinska Institutet. The p47 and p53 expression plasmids were a kind gift from Dr Pierre Hainaut.

\section{References}

1. Louis DN, Ohgaki H, Wiestler D and Cavenee WK: In: WHO classification of tumours of the central nervous system. Louis DN, Ohgaki H, Wiestler D, Cavenee WK (eds). IARC, Lyon, pp132-140, 2007.

2. Packer RJ and Vezina G: Management of and prognosis with medulloblastoma: therapy at a crossroads. Arch Neurol 65: 1419-1424, 2008.

3. Packer RJ: Childhood brain tumors: accomplishments and ongoing challenges. J Child Neurol 23: 1122-1127, 2008.
4. Gajjar A, Hernan R, Kocak M, et al: Clinical, histopathologic, and molecular markers of prognosis: toward a new disease risk stratification system for medulloblastoma. J Clin Oncol 22: 984-993, 2004.

5. Gilbertson R, Wickramasinghe C, Hernan R, et al: Clinical and molecular stratification of disease risk in medulloblastoma. Br J Cancer 85: 705-712, 2001.

6. Hemminki K, Tretli S, Sundquist J, Johannesen TB and Granstrom C: Familial risks in nervous-system tumours: a histology-specific analysis from Sweden and Norway. Lancet Oncol 10: 481-488, 2009.

7. Huang H, Mahler-Araujo BM, Sankila A, et al: APC mutations in sporadic medulloblastomas. Am J Pathol 156: 433-437, 2000.

8. Garre ML, Cama A, Bagnasco F, et al: Medulloblastoma variants: age-dependent occurrence and relation to Gorlin syndrome - a new clinical perspective. Clin Cancer Res 15: 2463-2471, 2009.

9. Tabori U, Baskin B, Shago M, et al: Universal poor survival in children with medulloblastoma harboring somatic TP53 mutations. J Clin Oncol (In press).

10. Pan E, Pellarin M, Holmes E, et al: Isochromosome $17 \mathrm{q}$ is a negative prognostic factor in poor-risk childhood medulloblastoma patients. Clin Cancer Res 11: 4733-4740, 2005.

11. Frank AJ, Hernan R, Hollander A, et al: The TP53-ARF tumor suppressor pathway is frequently disrupted in large/cell anaplastic medulloblastoma. Brain Res Mol Brain Res 121: 137-140, 2004.

12. Yakovleva T, Pramanik A, Terenius L, Ekstrom TJ and Bakalkin G: p53 latency-out of the blind alley. Trends Biochem Sci 27: 612-618, 2002.

13. Courtois S, Verhaegh G, North S, et al: DeltaN-p53, a natural isoform of p53 lacking the first transactivation domain, counteracts growth suppression by wild-type p53. Oncogene 21: 6722-6728, 2002.

14. Ghosh A, Stewart D and Matlashewski G: Regulation of human p53 activity and cell localization by alternative splicing. Mol Cell Biol 24: 7987-7997, 2004.

15. Maier B, Gluba W, Bernier B, et al: Modulation of mammalian life span by the short isoform of p53. Genes Dev 18: 306-319, 2004.

16. Yin Y, Stephen CW, Luciani MG and Fahraeus R: p53 stability and activity is regulated by Mdm2-mediated induction of alternative p53 translation products. Nat Cell Biol 4: 462-467, 2002.

17. Anensen N, Oyan AM, Bourdon JC, Kalland KH, Bruserud O and Gjertsen BT: A distinct p53 protein isoform signature reflects the onset of induction chemotherapy for acute myeloid leukemia. Clin Cancer Res 12: 3985-3992, 2006.

18. Baumbusch LO, Myhre S, Langerod A, et al: Expression of fulllength p53 and its isoform Deltap53 in breast carcinomas in relation to mutation status and clinical parameters. Mol Cancer 5: 47, 2006.

19. Boldrup L, Bourdon JC, Coates PJ, Sjostrom B and Nylander K: Expression of p53 isoforms in squamous cell carcinoma of the head and neck. Eur J Cancer 43: 617-623, 2007.

20. Bourdon JC, Fernandes K, Murray-Zmijewski F, et al: p53 isoforms can regulate p53 transcriptional activity. Genes Dev 19: 2122-2137, 2005.

21. Goldschneider D, Horvilleur E, Plassa LF, et al: Expression of C-terminal deleted p53 isoforms in neuroblastoma. Nucleic Acids Res 34: 5603-5612, 2006.

22. Friedman HS, Burger PC, Bigner SH, et al: Establishment and characterization of the human medulloblastoma cell line and transplantable xenograft D283. Med J Neuropathol Exp Neurol 44: 592-605, 1985.

23. Jacobsen PF, Jenkyn DJ and Papadimitriou JM: Establishment of a human medulloblastoma cell line and its heterotransplantation into nude mice. J Neuropathol Exp Neurol 44: 472-485, 1985.

24. He XM, Wikstrand CJ, Friedman HS, et al: Differentiation characteristics of newly established medulloblastoma cell lines (D384 Med, D425 Med, and D458 Med) and their transplantable xenografts. Lab Invest 64: 833-843, 1991.

25. Waha A, Koch A, Meyer-Puttlitz B, et al: Epigenetic silencing of the HIC-1 gene in human medulloblastomas. J Neuropathol Exp Neurol 62: 1192-1201, 2003.

26. Keles GE, Berger MS, Srinivasan J, Kolstoe DD, Bobola MS and Silber JR: Establishment and characterization of four human medulloblastoma-derived cell lines. Oncol Res 7: 493-503, 1995. 
27. Fults D, Pedone CA, Morse HG, Rose JW and McKay RD: Establishment and characterization of a human primitive neuroectodermal tumor cell line from the cerebral hemisphere. J Neuropathol Exp Neurol 51: 272-280, 1992.

28. Avery-Kiejda KA, Zhang XD, Adams LJ, et al: Small molecula weight variants of p53 are expressed in human melanoma cells and are induced by the DNA-damaging agent cisplatin. Clin Cancer Res 14: 1659-1668, 2008.

29. Oren M: Decision making by p53: life, death and cancer. Cell Death Differ 10: 431-442, 2003.

30. Xirodimas DP, Saville MK, Bourdon JC, Hay RT and Lane DP: Mdm2-mediated NEDD8 conjugation of p53 inhibits its transcriptional activity. Cell 118: 83-97, 2004.
31. Vogelstein B, Lane D and Levine AJ: Surfing the p53 network. Nature 408: 307-310, 2000.

32. Schuler M and Green DR: Transcription, apoptosis and p53: catch-22. Trends Genet 21: 182-187, 2005.

33. Chipuk JE, Kuwana T, Bouchier-Hayes L, et al: Direct activation of Bax by p53 mediates mitochondrial membrane permeabilization and apoptosis. Science 303: 1010-1014, 2004.

34. Marchenko ND, Zaika A and Moll UM: Death signal-induced localization of p53 protein to mitochondria. A potential role in apoptotic signaling. J Biol Chem 275: 16202-16212, 2000.

35. Moll UM, Wolff S, Speidel D and Deppert W: Transcriptionindependent pro-apoptotic functions of p53. Curr Opin Cell Biol 17: 631-636, 2005 . 